\section{Anos potenciais de vida perdidos por mulheres em idade fértil na cidade do Recife, Pernambuco, vítimas de morte por homicídio nos anos de 2001 e 2002}

\section{Years of potential life lost among female homicide victims of child-bearing age in the city of Recife, Pernambuco, (Brazil) in 2001 and 2002}

Marianne Weber Arnold ${ }^{1}$

Maria Arleide Silva ${ }^{2}$

Gilliatt Hanois Falbo Neto ${ }^{3}$

Rossana Paula Haimenis ${ }^{4}$

1-4 Diretoria de Pesquisa. Instituto Materno Infantil Professor Fernando Figueira-IMIP. Rua dos Coelhos, 300. Recife, PE, Brasil. CEP: 50. 070-550. E-mail: marianne@imip.org.br

\begin{abstract}
Objectives: to determine the frequency of death among women of child-bearing age, according to the main groups of basic causes of death; to calculate the Years of Potential Life Lost (YPLL) for homicide, comparing this index to the other most frequent causes of death; and to determine the YPLL coefficient for all four causes of death.

Methods: a descriptive, cross-sectional cohort study was carried out using the death certificates of women aged between 10 and 49 years in the city of Recife, State of Pernambuco, Brazil, in 2001 and 2002. The basic cause of death was classified according to the ICD-10; the YPLL was analyzed according to the criteria of the Brazilian Ministry of Health, as well as the Romeder-McWhinnie method, using the Epi-Info 2000 software package.

Results: neoplasm was the most frequent cause of death $(25.7 \%)$, followed by diseases of the circulatory system (21.2\%), external causes (17.6) and infectious/ parasitic diseases (9.6\%). Homicides were responsible for $49 \%$ (4380) of the total number of YPLL, with a YPLL coefficient of 8.82. The coefficient for breast cancer was 4.14 and for acute myocardial infarction 3.77 .

Conclusions: The risk of women losing potential years of life due to homicide was 2.1 times greater than the figure for breast cancer and 2.3 times greater than that for acute myocardial infarction.
\end{abstract}

Key words Potential years of life lost, Homicide, Mortality, Epidemiology, Violence against women

\section{Resumo}

Objetivos: determinar a freqüência dos óbitos de mulheres em idade fértil segundo os quatro principais grupos de causas básicas de óbito, calcular os Anos Potenciais de Vida Perdidos (APVP) para homicídio, comparando-o com as outras causas de morte mais freqüentes e os coeficientes de APVP destas quatro causas de óbito.

Métodos: estudo descritivo, tipo corte transversal, incluindo as declarações de óbito de mulheres com idade entre 10 e 49 anos, na cidade de Recife, Pernambuco, nos anos de 2001 a 2002. A causa básica de óbito foi classificada de acordo com a CID10, os APVP foram analisados segundo critérios do Ministério da Saúde e de Romeder e McWhinnie, utilizando-se o Epi-Info 2000.

Resultados: o grupo das neoplasias foi a causa de óbito mais freqüente (25,7\%), seguida das doenças do aparelho circulatório $(21,2 \%)$, causas externas $(17,6)$ e doença infecciosa e parasitária $(9,6 \%)$. Os homicídios foram responsáveis por 49\% (4380) do total de anos potenciais de vida perdidos, com um coeficiente de APVP de 8,82. O coeficiente de APVP para câncer de mama correspondeu a 4,14 e 3,77 para o infarto agudo do miocárdio.

Conclusões: o risco das mulheres perderem anos potenciais de vida por homicídio foi 2,1 vezes maior do que por câncer de mama e 2,3 do que por infarto agudo do miocárdio.

Palavras-chave Anos potenciais de vida perdidos, Homicídio, Mortalidade, Epidemiologia, Violência contra a mulher 


\section{Introdução}

Considerada como um problema de saúde pública desde a década de 90, a violência contra a mulher existe em todos os países e grupos sociais, indistintamente das condições socioeconômicas, credos e cultura. $^{1}$

Exercida em sua maioria no ambiente familiar e pelo parceiro íntimo, a violência contra a mulher se manifesta de formas e intensidades distintas, é um fenômeno complexo e tem múltipla causalidade. ${ }^{1-3}$ Suas consequiências vão desde seqüelas temporárias até a morte por homicídios.

No Brasil, Faúndes et al. ${ }^{4}$ investigaram mortes de mulheres de 10 a 49 anos em Campinas, São Paulo, as causas externas foram a principal causa de morte entre 10 e 34 anos e houve uma alta freqüência de morte por homicídios.

Albuquerque et al. ${ }^{5}$ investigaram em Recife, Pernambuco, as causas e fatores associados à mortalidade de mulheres em idade fértil encontrando que $32,2 \%$ das mortes tiveram com causa o homicídio, correspondendo à terceira causa de morte na população do estudo.

As taxas de mortes prematuras têm se elevado com o recrudescimento da violência. Assim, os Anos Potenciais de Vida Perdidos (APVP), é um indicador que estima o tempo que a pessoa deveria ter vivido se não morresse prematuramente, tem sido a medida utilizada para o monitoramento dessas mortes e para a redefinição das prioridades em saúde pública. A medida dos APVP enfatiza as causas específicas de óbito que afetam grupos etários mais jovens, resultando numa ordenação diferente das causas de morte. ${ }^{6-, 8}$

O APVP pode servir como medida resumo, sendo mais fácil de ser compreendida e comparada entre populações do que um grande número de coeficientes específicos por idade, prestando-se para uma triagem inicial na análise de dados em áreas que apresentam excesso de mortalidade. ${ }^{9}$

Haensel $^{10}$ propôs o uso de coeficientes padronizados de APVP com o objetivo de realizar comparações entre diferentes áreas e tempos, e uma idade padrão como limite superior de cálculo, tendo em vista que a expectativa de vida ao nascer varia de acordo com cada região e no decorrer dos anos, sendo então de difícil poder comparativo.

Considerando a importância crescente de mortes de mulheres em idade reprodutiva, realizou-se uma análise da mortalidade de mulheres com idade entre 10 e 49 anos na cidade de Recife, por um período de dois anos (2001 a 2002). Os objetivos foram: determinar a frequiência dos óbitos de mulheres em idade fértil segundo os quatro principais grupos de causas básicas de óbito, calcular os APVP para homicídio, comparando-o com as outras três causas de morte mais freqüentes e determinar os coeficientes de APVP destas quatro principais causas de óbito.

\section{Métodos}

Foi realizado um estudo descritivo, tipo corte transversal, incluindo-se todas as Declarações de Óbito (DO) de mulheres entre 10 e 49 anos residentes no Recife, Pernambuco, mortas no período entre $1^{\circ}$ de janeiro de 2000 a 31 de dezembro de 2001. As informações foram obtidas automaticamente do banco de dados da Diretoria de Epidemiologia e Vigilância à Saúde (DIEVS) da Secretaria de Saúde da Prefeitura da Cidade do Recife.

A causa básica do óbito foi codificada de acordo com a Classificação Estatística Internacional de Doenças e Problemas Relacionados à Saúde, 10. revisão, (CID-10) $)^{11}$ e analisadas no software EpiInfo 2000.

Para obtenção dos APVP foi feita a distribuição dos óbitos por agrupamento de idade. Em seguida multiplicou-se o número de óbitos em cada intervalo de idade pelo número de anos que faltavam para atingir a idade limite de 70 anos. Essa diferença é obtida a partir do ponto médio de cada faixa etária. A soma desses produtos fornece o total de APVP, valor que representa o número estimado de perdas para uma causa específica ou para todas as causas. ${ }^{12}$

Adotou-se no presente estudo os critérios sugeridos pelo Ministério da Saúde, que estabelece uma idade limite para o cálculo dos APVP em 70 $\operatorname{anos}^{13}$ e a técnica aplicada foi a proposta por Romeder e McWhinnie ${ }^{12}$ que estabelecem uma idade limite para o cálculo dos APVP com base na vida média da população. Foram utilizados para base de cálculo coeficiente dos APVP, os dados do Instituto Brasileiro de Geografia e Estatística (IBGE), que no ano 2000 contabilizou 496.210 mulheres com idade entre 10 e 49 anos. ${ }^{14}$

$\mathrm{O}$ acesso ao do banco de dados do Serviço de Informação de Mortalidade (SIM) foi autorizada pela DIEVS da Secretaria de Saúde da Prefeitura da Cidade do Recife, e o estudo foi aprovado pelo Comitê de Ética em Pesquisa em Seres Humanos do Instituto Materno Infantil Prof. Fernando Figueira-IMIP.

\section{Resultados}

A distribuição de freqüência dos óbitos mostra que o 
grupo das neoplasias foi a principal causa de óbito em mulheres com idade entre 10 a 49 anos na cidade do Recife nos anos investigados, representando $25,7 \%$ do total de óbitos. Dentre as neoplasias o câncer de mama foi o mais freqüente, com 73 dos 308 casos $(23,7 \%)$. O segundo grupo foi o das doenças do aparelho circulatório, com 254 óbitos $(21,2 \%)$ destes, $69(27,1 \%)$ por infarto agudo do miocárdio (IAM). Os grupos das causas externas e das doenças infecciosas e parasitárias ocuparam a terceira e quarta posição representando respectivamente $17,6 \%$ e $9,6 \%$ dos casos. A tuberculose pulmonar foi responsável por 27 mortes dentre os 116 óbitos de doenças infecciosas e parasitárias (Tabela 1).

A Tabela 2 mostra a distribuição dos APVP por homicídio e das outras quatro causas básicas mais frequientes de óbito das mulheres em idade fértil em 2001 e 2002. As faixas de idade entre 10-19 e 20-29 apresentaram os maiores valores de APVP (1595 e
1620 respectivamente) dentre as vítimas de homicídio. O câncer de mama foi responsável pela perda de 2075 anos potenciais de vida, destes, 1250 estavam com idade entre 40 a 49 anos. Mesmo não havendo morte em menores de 30 anos entre as que faleceram por infarto agudo do miocárdio, essa causa somou um total de 1875 anos de vida perdidos. A faixa de idade entre 40-49 anos apresentou o maior número de APVP pelas quatro causas mais freqüentes no grupo estudado, com 4075 APVP $(29,4 \%)$. Em relação ao homicídio, essa causa foi a maior responsável pelos APVP para as faixas de idade de 10-19, 20-29 e 30-39.

Os coeficientes de APVP por homicídio foram de 8,82 e de 5,56 para os acidentes. O coeficiente de APVP para câncer de mama correspondeu a 4,14 e foi de 3,77 para o infarto agudo do miocárdio. Os dados revelam que os APVP por homicídio são 2,1 vezes maiores do que por câncer de mama e 2,3 do que por infarto agudo do miocárdio (Tabela 3 ).

\section{Tabela 1}

Distribuição de freqüência dos óbitos de mulheres em idade fértil, segundo as quatro principais causas básicas de óbito. Recife, Pernambuco, 2001-2002.

\begin{tabular}{lcc}
\hline Causa básica & N & $\%$ \\
\hline Neoplasia & 308 & 25,7 \\
Doenças do aparelho circulatório & 254 & 21,2 \\
Causas externas & 211 & 17,6 \\
Doença infecciosa e parasitária & 116 & 9,6 \\
Demais causas & 308 & 25,7 \\
Total & 1197 & 100,0 \\
\hline
\end{tabular}

Tabela 2

Distribuição dos anos potenciais de vida perdidos segundo as quatro causas básicas de óbito mais freqüentes por faixa etária de mulheres em idade fértil. Recife, Pernambuco, 2001-2002.

\begin{tabular}{|c|c|c|c|c|c|c|c|c|c|c|}
\hline \multirow[b]{2}{*}{ Idade } & \multicolumn{2}{|c|}{ Homicídio } & \multicolumn{2}{|c|}{ Acidentes } & \multicolumn{2}{|c|}{ Câncer de mama } & \multicolumn{2}{|c|}{ Infarto agudo do miocárdio } & \multicolumn{2}{|c|}{ Total } \\
\hline & APVP & $\%$ & APVP & $\%$ & APVP & $\%$ & APVP & $\%$ & APVP & $\%$ \\
\hline $10-19$ & 1595 & 11,5 & 1210 & 8,7 & - & - & - & - & 3135 & 22,6 \\
\hline $20-29$ & 1620 & 11,7 & 765 & 5,5 & 90 & 0,6 & - & - & 3285 & 23,7 \\
\hline $30-39$ & 840 & 6,0 & 385 & 2,7 & 735 & 5,3 & 525 & 3,7 & 3325 & 24,0 \\
\hline $40-49$ & 325 & 2,3 & 400 & 2,8 & 1250 & 9,0 & 1350 & 9,8 & 4075 & 29,4 \\
\hline Total & 4380 & 31,6 & 2760 & 20 & 2075 & 15 & 1875 & 14 & 13820 & 100,0 \\
\hline
\end{tabular}

APVP=Anos potenciais de vida perdidos.

Fonte: Diretoria de Epidemiologia e Vigilância à Saúde (DIEVS) da Secretaria de Saúde da Prefeitura da Cidade do Recife. 
Tabela 3

Coeficientes de anos potenciais de vida perdidos das quatro principais causas básicas de óbito de mulheres em idade fértil. Recife, Pernambuco, 2001-2002.

\begin{tabular}{lc}
\hline Causa básica & Coeficiente de Anos Potenciais de Vida Perdidos \\
\hline Homicídio & 8,82 \\
Acidentes & 5,56 \\
Câncer de mama & 4,14 \\
Infarto agudo do miocárdio & 3,77 \\
\hline
\end{tabular}

Fonte: Diretoria de Epidemiologia e Vigilância à Saúde (DIEVS) da Secretaria de Saúde da Prefeitura da Cidade do Recife.

\section{Discussão}

No presente estudo, os achados relativos às causas externas ocuparam o terceiro lugar dentre as causas de óbitos de mulheres em idade fértil, resultados semelhantes foram encontrados em outros estudos de análises de óbitos em várias regiões do Brasil. ${ }^{4,5,15-17}$

Dados do National Center for Health Statistics (NCHS), ${ }^{18}$ de 2001, também evidenciaram as causas externas como principal causa de morte entre mulheres em idade fértil nos Estados Unidos da América.

Na população estudada, as neoplasias tiveram maior frequiência entre as causas de morte. Esse dado pode apontar para a precariedade das ações preventivas, seja na educação em saúde da população ou pela ausência de equipamentos de saúde que atendam a demanda, contribuindo assim para o abandono dos tratamentos, ou devido à baixa condição socioeconômica da população que muitas vezes impossibilita a realização de exames periódicos.

A elevada freqüência de mortes violentas encontrada no nosso estudo, destacando-se os homicídios que chegaram quase a $50 \%$ dos óbitos por causas externas, são resultados com números superiores aos de Albuquerque et al. ${ }^{5}$ que encontraram um terço de mortes por homicídios em mulheres em idade fértil no ano de 1992/1993. Esses achados podem ser conseqüências das mudanças no comportamento social da mulher, que há décadas vem ocupando espaços antes exclusivos ao homem. Entretanto, tal motivo não pode por si só justificar os achados, haja vista a múltipla causalidade das práticas violentas contra a mulher.

Ao utilizar o indicador anos potenciais de vida perdidos na análise dessas mortes, o homicídio passou a ocupar o primeiro lugar, seguido dos acidentes, que juntos representaram a perda de 7140 $\operatorname{APVP}(51,6 \%)$ dentre as quatro principais causas básicas de óbito. O coeficiente de APVP demonstrou que a estimativa de perder anos potenciais de vida por homicídio foi aproximadamente duas vezes maior que por câncer de mama e 2,3 vezes maior que o risco de perder anos potencias de vida por IAM. Estes dados são semelhantes aos encontrados em outros estudos brasileiros, onde os acidentes e homicídios foram também as principais causas de APVP ${ }^{19,20}$ porém, nesses estudos, a principal causa de APVP foram os acidentes,e, em Recife o homicídio ficou em primeiro lugar.

Em outro estudo realizado no Recife, avaliando os anos potenciais de vida perdidos por crianças e adolescentes entre um mês e 19 anos para os óbitos do ano de 1997, o impacto dos homicídios foi ainda mais importante, sendo responsável por 13.965 APVP (59\% do total), com um coeficiente de 27,20 anos potenciais de vida perdidos, que demonstram que a estimativa de perder anos potenciais de vida por homicídio foi aproximadamente três vezes maior do que por pneumonias, que foi a segunda causa de APVP no grupo estudado. ${ }^{21}$

Os APVPs, como já referido, é considerado um indicador de saúde. Realizar esae cálculo permite visualizar o impacto econômico e social causado em sua grande parte por mortes evitáveis e prematuras. Constatar-se o grande prejuízo em anos de vida precocemente ceifados pela violência deve alertarnos para necessidades urgentes de prevenção tanto no âmbito social e político, como na saúde da população.

Recife é uma capital sabidamente violenta, onde o número de homicídios de grupos vulneráveis tais como as crianças, adolescentes e mulheres têm aumentado assustadoramente. Estudos como os que têm sido realizados na tentativa de conhecer melhor 
as mais diferentes facetas desse quadro dramático são fundamentais para o estabelecimento de políticas públicas que possam frear esta escalada dos índices

\section{Referências}

1. Krug EG, Dahlberg LL, Mercy JÁ, Zwi A, Lozano-Ascencio A. Rapport Mondial sur la Violence et la Santé. Géneve. WHO; 2002. Disponível em URL: www.who.int [2007 20 Sept]

2. WHO (World Health Organization). Relatório mundial de saúde. Géneve; 2002.

3. Figueira MCS. Prevalência de morte de mulheres em idade fértil por causas externas na cidade do Recife: 2001/2002 [dissertação mestrado]. Recife: Instituto Materno Infantil de Pernambuco; 2004.

4. Faundes A, Parpineli MA, Cecatti JG. Mortalidade de mulheres em idade fértil, em Campinas, São Paulo 19851994. Cad Saúde Pública. 2000; 16: 671-9.

5. Albuquerque RM, Cecatti JG, Hardy EE, Faundes A. Causas e fatores associados à mortalidade de mulheres em idade reprodutiva, Recife, Brasil. Cad Saúde Pública. 1998; 14: 41-8.

6. Gardner JW, Sanborn JS. Years of potential life lost (YPLL)what does it measure? Epidemiology. 1990; 1: 322-9.

7. Lira MMTA. Mortalidade prematura no município de São Paulo; anos potenciais de vida perdidos: 1980, 1985, 1990 e 1995 [dissertação mestrado]. São Paulo: Faculdade de Saúde Pública da Universidade de São Paulo; 1998.

8. McDonnell S, Vossberg K, Hopkins RS, Mittan B. Using YPLL in health planning. Public Health. 1998; 113: 55-61.

9. Kleinman JC. Age-adjusted mortality indexes for small areas: applications to health planning. Am J Public Health. 1977; 67: 834-80.

10. Haenszel W. A standardized rate for mortality defined in units of lost years of life. Am J Public Health. 1950; 40: 1726.

11. OMS (Organização Mundial da Saúde). Classificação Estatística Internacional de Doenças e Problemas Relacionados à Saúde: 10. revisão. 10. ed. São Paulo: Centro da OMS para Classificação das Doenças em Português; 1999.

12. Romeder JM, Mcwhinnie JR. The development of potential years of life lost as an indicator of premature mortality. Rev Epidemiol Sante Publique. 1978; 26: 97-115. de violência, principalmente na sua forma mais cruel, que é o homicídio.

13. Brasil. Ministério da Saúde. Investigação sobre perfis de saúde. Brasília, DF; 1998.

14. IBGE (Instituto Brasileiro de Geografia e Estatística). Resultados da amostra do censo demográfico 2000. Disponível em http://www.ibge.gov.br/cidadesat/ default.php [2007 set 14].

15. Haddad N, Silva MB. Female mortality in reproductive age in the State of São Paulo, Brazil, 1991-1995: underlying causes of death and maternal mortality. Rev Saúde Pública. 2000; 34: 64-70.

16. Reis AC, Souza ER, Minayo MCS, Malaquias JV. Mortalidade feminina por causas externas: Brasil e macro regiões. Rio de Janeiro: Centro Latino Americano de Estudos de Violência e Saúde Jorge Careli; 2002.

17. Minayo MCS, Souza ER, Silva CMFP. Perfil da mortalidade por causas externas no Brasil: análise temporal das décadas de 80 e 90. Brasília, DF: Ministério da Saúde; 2001.

18. NCHS (National Center for Health Statistics). Safer healthier people: 10 leading causes of death, United States2001, all races, female. Atlanta; 2004.

19. Silva MGC. Anos Potenciais de Vida Perdidos por causas evitáveis, segundo sexo em Fortaleza, em 1996-1998. Epidemiol Serv Saúde. 2003; 12: 100-10.

20. Cortes H, Peixoto G, Souza ML. O indicador Anos Potenciais de Vida Perdidos e a ordenação das mortes em Santa Catarina. Disponível em URL: http://www.saude.sc. gov.br/ gestores/sala_de_leitura/artigos/Mortalidade/ apvp1.pdf [2004 $12 \mathrm{dez}$ ].

21. Arnold MW, Falbo Neto GH, Figueiroa JN. Years of potential life lost by children and adolescent victims of homicide: Recife, 1997. J Trop Pediatr. 2002; 48: 67-71. 